\title{
Can Quantum Analogies Help Us to Understand the Process of Thought?
}

\author{
Paavo Pylkkänen \\ Department of Cognitive Neuroscience and Philosophy \\ University of Skövde, Sweden \\ and \\ Department of Philosophy, History, Culture and Art Studies \\ University of Helsinki, Finland
}

\begin{abstract}
A number of researchers today make an appeal to quantum physics when trying to develop a satisfactory account of the mind, an appeal still felt to be controversial by many. Often these "quantum approaches" try to explain some well-known features of conscious experience (or mental processes more generally), thus using quantum physics to enrich the explanatory framework or explanans used in consciousness studies and cognitive science. This paper considers the less studied question of whether quantum physical intuitions could help us to draw attention to new or neglected aspects of the mind in introspection, and in this way change our view about what needs explanation in the first place. Although prima facie implausible, it is suggested that this could happen, for example, if there were analogies between quantum processes and mental processes (e.g., the process of thinking). The naive idea is that such analogies would help us to see mental processes and conscious experience in a new way.

It has indeed been proposed long ago that such analogies exist, and this paper first focuses at some length on David Bohm's formulation of them from 1951. It then briefly considers these analogies in relation to Smolensky's more recent analogies between cognitive science and physics, and Pylkkö's aconceptual view of the mind. Finally, Bohm's early analogies will be briefly considered in relation to the analogies between quantum processes and the mind he proposed in his later work.
\end{abstract}

\section{Introduction}

There are by now many approaches that seek to explain conscious experience, and mental processes more generally, in terms of a conceptual framework involving ideas from quantum physics (see e.g. Atmanspacher 2011 and the references therein). In this paper I will explore another, less studied possibility, namely that quantum physics might not only help us 
to explain commonly acknowledged features of the mind, but also to draw attention to neglected but important aspects of our inner experience. In other words, I am interested in the question of whether the consideration of quantum physics can change our view of what there is to be explained about the mind in the first place (the explanandum), over and above the more obvious role it might play as affecting the explanans, i.e. the conceptual framework with which we try to explain some well-known features of conscious experience and mental processes (cf. van Gulick 1995).

Insofar as a quantum physical framework is an explanans that helps us to see mind/consciousness as the explanandum in the new way, we could say that "explanandum is explanans-laden". Van Gulick (1995) does not consider this possibility in his interesting paper "What would count as explaining consciousness?", although he makes otherwise a very useful attempt to "divide and conquer" the problem of consciousness. But if we want to acknowledge at the outset any possible bias in our attempts to explain conscious experience and mental processes, then we ought to consider not only how our explanans deals with the explanandum, but also the more difficult issue of whether the explanans we always already have affects the way we perceive and define the explanandum in the first place. ${ }^{1}$

In consciousness studies, introspection plays a central role as a method. But when studying conscious experience in introspection, how much is what we "see" affected by the scientific theory and, more generally, the worldview or paradigm we happen to hold? To what extent is introspection theory- or paradigm-laden?

To unpack the idea of a "paradigm-ladeness of introspection", the first point to note is that in the philosophy of science it is a household fact that "observation is theory-laden", that what we observe in general, and also in our scientific experiments, is affected by the theory we are using, and other assumptions, and our (more or less unconsciously held) Weltanschauung or paradigm (see e.g. Hanson (1958), Kuhn (1962/1970), and Feyerabend (1975/1993); see also Suppe (1977)). A nice summary

\footnotetext{
${ }^{1}$ In both analytical and (especially) continental philosophy since the middle of the last century it has been one of leading paradigms to think that "... our experiential access to reality is essentially mediated by the historical contexts of meaning - languages, concepts, norms - in which we live, and which determine in advance what we may see and identify as meaningful phenomena" (Westerlund 2014, p. 2). At the end of his recent extensive study of Heidegger's account of the problem of phenomenality, Westerlund (2014, p. 506) makes some proposals that are very relevant to our present concerns: "We always already live in historically transmitted and collectively sanctioned concepts and meanings which tend to guide our sight and which invite us to attend only to what our pre-understanding commands." Indeed. The basic idea of the present paper is that the prevailing mechanistic spirit that has been much strengthened by classical physics tends to lead us to ignore important aspects of our experience (cf. the criticism of the dominant role of the "containment metaphor" and its accompanying ontology of little things and "microbangings" in contemporary analytical metaphysics by Ladyman and Ross (2007)).
} 
of the Feyerabend-Kuhn view of theory-ladeness is given by Suppe (1977, p. 689):

[Shapere] finds that the Feyerabend-Kuhn view makes the following chain of inferences:

(i) Observation, if it is to be relevant, must be interpreted.

(ii) That in terms of which interpretation is made is always theory.

(iii) The theory that interprets is the theory to be tested.

(iv) The theory to be tested is "the whole of science" (or a branch thereof).

(v) This whole forms a unity ("paradigm" or "high-level background theory").

(vi) This unified whole not only serves as a basis of interpretation, but also determines ("defines") what counts as an observation, problem, method, solution, and so forth.

For the purposes of this paper the question is whether it makes a difference to consciousness studies (and introspection in particular) that "the whole of science" includes quantum physics. In other words, can the radically different "whole of science" we get as a result of quantum physics affect the way we interpret our observations in introspection? Can it affect it so that we can obtain in some ways a more complete and accurate explanation of conscious experience and mental processes?

Note that the Feyerabend-Kuhn approach raises the issues of relativism and circularity in a powerful way. If the theory we are allegedly testing is also the one we use to interpret the results, and the one which defines what counts as observation, problem, method and solution, how objective, neutral and impartial can such testing be judged to be? There is a risk of a deep circularity that arises if we take seriously the idea that observation - including introspection - is theory-laden.

In this paper, however, I will not try to tackle the difficulties raised by circularity and relativism. For the sake of the argument, I will assume that there is a sense in which a given theory might give us a more complete and more accurate description and explanation of a given phenomenon or domain than some other theory. And thus, I assume that it is at least in principle possible that, for example, a scientific worldview taking into account the results of new quantum physics could help us to describe and explain the mind in a better way than the common-sense worldview of classical physics. Let us now proceed to explore in more detail how this might be possible.

It seems fairly obvious that people who are familiar with quantum physics develop a whole new set of intuitions about, for example, what it can mean for a phenomenon to be physical, or about general principles that prevail in phenomena. The possibility to be explored here then is that, when studying conscious experience in introspection, these people 
might well make use of these new intuitions and see new aspects of the mind, or give importance to aspects that others in a sense see but tend to neglect.

If introspection is theory-laden, then people who hold the classical, mechanistic worldview as the only relevant truth might well tend to see and emphasize only the classical and mechanistic aspects of conscious experience and mental processes, while people equipped with classical as well as quantum intuitions might see broader aspects. The "classical and mechanistic" aspects I have in mind are separable objects in experience, causal relations between them, the idea that (more or less) Euclidian-Newtonian spacetime is the arena where conscious experience takes place. In the domain of thought and language, some cognitive scientists emphasize that thinking is essentially mechanical symbol manipulation. Presumably they find such characterization introspectively accurate.

I do not claim that when we ordinarily and pre-theoretically introspect we would automatically find only such mechanistic features. Rather, the idea is that much of, say, contemporary philosophy of mind and cognitive (neuro)science involves strongly mechanistic assumptions that direct us to draw attention to the mechanistic aspects of the mind, while making it more difficult to see other aspects. When you have a hammer in your hand, everything in the world looks like a nail. Analogously, to an introspector equipped with the mechanistic conceptual tools of modern cognitive science and philosophy of mind, conscious experience and mental processes may well look more mechanistic than to someone with a different, less mechanistic paradigm. This does not, of course, mean that the mind has no mechanistic aspects. But it should make us more open to the possibility that the mind has also other kinds of aspects that might be better appreciated in the light of a different theory.

The above, if correct, suggests an interesting way in which quantum approaches to consciousness and mental processes can be relevant. They might draw attention to important aspects of the mind that tend to be neglected (or simply "not seen") by the prevalent more mechanistic approaches to the mind. This may sound prima facie quite implausible. How on earth could quantum physics, which deals with atomic phenomena, help us to more accurately introspect conscious experience and mental processes, which appear to be completely different phenomena at a different, perhaps neurobiological level of organization (as, e.g., Revonsuo (2006) assumes)?

One way in which this could be imagined is in terms of analogies between quantum phenomena and mental phenomena. ${ }^{2}$ Suppose, for the

\footnotetext{
${ }^{2}$ For an interesting paper discussing analogies between modern physics and cognitive psychology, see Shanon (1991). For a defense of the role of analogy in scientific reasoning, see Campbell (1957) and Hesse (1966, 1974); see also Pickering (1984) and Cushing (1990), all quoted in Guarini (2003).
} 
sake of the argument, that quantum phenomena and some features of our inner experiences resemble each other in some important respects. For example, let us assume that the way conscious thought typically "proceeds", or changes its state from moment to moment, would resemble the way a quantum system typically moves. Then suppose that someone familiar with quantum processes would begin to systematically consider the nature of their inner experience and thought processes. Seeing resemblances between the two domains might well constitute a non-trivial, exciting discovery.

Now, it so happens that many physicists have in fact proposed that there are strong resemblances between quantum processes and our inner experiences and thought processes. Such analogies were proposed to exist early on by the founding figures of quantum theory, for example by Niels Bohr (1934). In contemporary research similar analogies still play an important role. Consider, for example, Globus' (2003) interesting suggestion that Vitiello's dual-mode quantum brain dynamics resembles Heidegger's dynamical Ereignis whose modes are "being" and "time".

In this paper I will focus upon a fairly detailed early discussion of analogies between quantum and thought processes put forward by the physicist-philosopher David Bohm (1951). Interestingly in view of the questions we started off with, Bohm's analogies seem to draw attention to certain aspects of the mind that, although at least potentially fundamental and important when noticed and considered, nevertheless tend to be neglected in many contemporary academic studies of the mind.

In this paper my aim is thus, via considering Bohm's analogies, to explore whether quantum intuitions can help us to understand conscious experience and mental processes in a new and better way - better in the sense that such quantum intuitions would draw attention to and help to explain certain important but neglected characteristics of the mind. In order to realize that aim I will first describe and discuss at some length Bohm's analogies between quantum processes and thought processes; I will then consider these in relation to Smolensky's (1988) analogies between physics and cognitive science; I next interpret Bohm's analogies in terms of Pylkkö's (1998) aconceptual view of the mind; and in my concluding reflections I briefly consider some of Bohm's later interpretations of quantum theory and the way he used them to develop new analogies to understand the mind.

\section{Analogies Between Quantum Processes and Thought Processes}

Already the founding figures of quantum theory, and Niels Bohr in particular, drew attention to the possible relevance of quantum physics 
to our understanding of the mind (see, e.g., Bohr 1934). A particularly clear early statement about certain close resemblances between quantum processes and thought processes, influenced by Bohr's ideas, can also be found in Bohm's acclaimed 1951 textbook Quantum Theory. That book, written when Bohm was still an advocate of the so called "orthodox" or "Copenhagen" interpretation of quantum theory, puts a strong emphasis on the physical meaning of quantum theory, as opposed to its mathematical formalism, which is focused at in many textbooks. ${ }^{3}$

When he wrote his 646-page book Bohm thus certainly had the more general physical and philosophical significance of quantum physics strongly in his mind (see especially Chap. 8, "An attempt to build a physical picture of the quantum nature of matter", pp. 144-172). This put him into a good position to consider "wide ranges of experience in which occur phenomena possessing striking resemblances to quantum phenomena". His basic claim in this regard was that there is a close analogy between quantum processes and our inner experiences and thought processes. After discussing such analogies he also provided some speculations of the underlying reasons for the existence of the analogies (pp. 168-172).

Let us now consider Bohm's discussion in some detail. I have included fairly long quotations and added explanatory comments in order to make the paper more accessible to those without a strong background in quantum physics. Also, I let Bohm himself speak on the physics issues whenever this seems reasonable. A closer examination of his 1951 analogies is also useful from the point of view of understanding the historical roots of the idea that quantum physics might play an important role in the study of the mind.

\subsection{An Uncertainty Principle for the Process of Thought}

Bohm (1951) starts off by considering the uncertainty principle of quantum theory and certain aspects of our thought processes (p. 169):

If a person tries to observe what he is thinking about at the very moment that he is reflecting on a particular subject, it is generally agreed that he introduces unpredictable and uncontrollable changes in the way his thoughts proceed thereafter. Why this happens is not definitely known at present ... If we compare (1) the instantaneous

\footnotetext{
${ }^{3}$ It has turned out that while Bohm initially thought that he was presenting Bohr's interpretation of quantum theory, his account is actually closer to Pauli's interpretation (Bohm in discussion with M. Wilkins, The David Bohm Papers, Birkbeck College, London, www.bbk.ac.uk/lib/about/bohm). This explains some of the responses to his book that Bohm received from Bohr, Pauli and Einstein. Bohr remained silent while Pauli sent an enthusiastic reply. According to Bohm, Einstein liked the book but was still not happy with the usual interpretation of quantum theory and wanted to discuss the matter with Bohm. These discussions had a key motivating role for Bohm's formulation of his 1952 non-local "hidden-variables" approach.
} 
state of a thought with the position of a particle and (2) the general direction of change of that thought with the particle's momentum, we have a strong analogy.

In classical, Newtonian physics one can, in principle, measure the momentum and position of a particle accurately at the same time - a special case of that is when we look at a stationary object where momentum equals zero and the position is where we see and measure it to be located. At the quantum level it is not possible to measure position and momentum simultaneously with arbitrarily high accuracy, beyond the limits set by the uncertainty principle. Bohm implies that in this respect thought is more quantum-like than classical-like. Given that the uncertainty principle is one of the most characteristic features of quantum physics (and relates to Bohr's principle of "complementarity", see Plotnitsky 2010), it is at least prima facie interesting whether or not a kind of uncertainty principle (and thus complementarity) also applies to aspects of our thought process.

Bohm (1951, p. 169) continues:

... a person can always describe approximately what he is thinking about without introducing significant disturbances in his train of thought. But as he tries to make the description precise, he discovers that either the subject of his thoughts or their trend or sometimes both become very different from what they were before he tried to observe them. Thus, the actions involved in making any single aspect of the thought process definite appear to introduce unpredictable and uncontrollable changes in other equally significant aspects.

This, again, is reminiscent of quantum physics. It is possible to make "unsharp measurements" where one obtains an approximate idea of the position of a particle, without making the momentum completely unknown. But should one want to measure the position precisely, the momentum becomes undefined. Thus, even if we were able to make "unsharp measurements" of both the direction and content of our thought process at a given instant, this would be analogous to measurements in quantum physics.

\subsection{Holistic Features of Thought and Quantum Processes}

Bohm further develops the analogy by suggesting that the "significance of thought processes" appears to be characterized by indivisibility (p. 169):

... if a person attempts to apply to his thinking more and more precisely defined elements, he eventually reaches a stage where further analysis cannot even be given a meaning. Part of the significance of each element of a thought process appears, therefore, to originate in its indivisible and incompletely controllable connections with other elements. 
In a footnote, he adds:

Similarly, part of the connotation of a word depends on the words it is associated with, and in a way that is not, in practice, completely predictable or controllable (especially in speech). In fact, the analysis of language, as actually used, into distinct elements with precisely defined relations between them is probably impossible.

Bohm is here concerned with the nature of meaning. We may customarily think that elements of our thought and language, such as sentences or words, carry their meanings autonomously. Just as classical physics assumes that the physical world consists of some basic elements (particles and fields), whose "intrinsic nature" is not affected by the relationships they enter, so we might assume that thought and language can be analyzed to some basic elements which have determinate and well-defined meanings, independently of the relations that such elements have to others or to their surrounding context.

The idea of "elementary propositions" and "names" in Wittgenstein's Tractatus can perhaps be seen as an attempt to conceive language in such a way. Wittgenstein writes (4.221 in Tractatus, quoted in Jones (1975, p. 204)): "It is obvious that the analysis of propositions must bring us to elementary propositions which consist of names in immediate combination". Jones (1975, p. 204) further describes Wittgenstein's view as follows: "... unless a sentence can be analyzed into a series of simple symbols ('primitive names'), each of which refers to a simple object that can be 'elucidated' by primitive propositions, the sentence is meaningless".

As is well known, the later Wittgenstein gave up such an atomistic view of meaning and emphasized, for example, that to determine the meaning of a term we ought to consider how the term is used. It is obvious that Bohm likewise did not think that the structure of thought and language is atomistic. Instead, he emphasized the holistic nature of meaning. Elements of our thought process and language do not have their meanings autonomously, but they originate in the connections with other elements. And, Bohm suggests, these connections are both indivisible and incompletely controllable. For him this implies that it is not possible to analyze language beyond a certain stage and expect to find elements with well-defined significance.

Quine, as is well known, also emphasized the holistic nature of language. He held that we cannot define concepts and words individually, for language is a holistic system. Quine has, following Duhem, famously analyzed the implications of this holism for the empirical verification of propositions (Quine 1951/1961, p. 41-43):

... our statements about the external world face the tribunal of sense experience not individually but only as a corporate body. ... 
The idea of defining a symbol in use was ... an advance over the impossible term-by-term empiricism of Locke and Hume. The statement, rather than the term, came with Bentham to be recognized as the unit accountable to an empiricist critique. But what I am now urging is that even in taking the statement as unit we have drawn our grid too finely. The unit of empirical significance is the whole of science.

Any statement can be held true come what may, if we make drastic enough adjustments elsewhere in the system.

These brief quotations provide some broader context for Bohm's ideas about the indivisibility of meaning. Interestingly, Bohm's remarks were published in the very same year, 1951, when Quine published his famous article "Two Dogmas of Empiricism", from which the above quotations are taken. Of course, Bohm is not specifically concerned with propositional thought or truth conditions, but more with meaning as we encounter it with actual thought processes and actual language use. Bohm's way of looking at meaning and thought as psychological phenomena seems more similar to, for example, Gestalt psychology, (see, e.g., Sundqvist 2003, pp. 177-181) than to analytical philosophy of language which is more concerned with logical and semantic properties of language.

It is an interesting question whether the holism of thought and language, seen as an actual psychological phenomenon, is connected with the holism of semantic properties of propositions (which some see as mindindependent). This question, however, will not be pursued here. Instead, let us go on to consider in what way Bohm thinks that there is an analogy between the holistic features of thought/language and quantum processes (Bohm 1951, p. 169):

Similarly, some of the characteristic properties of a quantum system (for example, wave or particle nature) depend on indivisible and incompletely controllable quantum connections with surrounding objects. Thus, thought processes and quantum systems are analogous in that they cannot be analyzed too much in terms of distinct elements, because the "intrinsic nature" of each element is not a property existing separately from and independently of other elements but is, instead, a property that arises partially from its relation with other elements. In both cases, an analysis into distinct elements is correct only if it is so approximate that no significant alteration of the various indivisibly connected parts would result from it.

To get a better idea of the quantum physical side of the analogy, it is useful to consider another description of indivisible quantum connections that Bohm gives. At the quantum level of accuracy, he says (p. 166)

... the quanta connecting object and environment constitute irreducible links that belong, at all times, as much to one part as to the 
other. Since the behavior of each part depends as much on these quanta as on its "own" properties, it is clear that no part of the system can be thought of as separate.

If, in a classical experiment, we discovered the presence of irreducible "links" between objects, we should then postulate a third object, the link, and thus re-establish the old type of description [analysis into parts], this time in terms of three parts to the system. In quantum theory, however, these quanta do not constitute separate objects, but are only a way of talking about indivisible transitions of the objects already in existence.

Consider, for example a situation in which a hydrogen atom in the ground state absorbs a quantum of energy from an electromagnetic field (p. 166-167): "during the process of transition, both systems are coupled because they are exchanging an indivisible quantum of energy belonging as much to the electron as to the electromagnetic field." More generally, because strictly speaking all physical "parts" that interact with each other are connected by such indivisible quanta to other "parts", quantum theory implies a fundamentally holistic view of the physical universe. Bohm (p. 167) concludes that

the entire universe must, on a very accurate level, be regarded as a single indivisible unit in which separate parts appear as idealizations permissible only on a classical level of accuracy of description. This means that the view of the world as being analogous to a huge machine, the predominant view from the sixteenth to nineteenth centuries, is now shown to be only approximately correct. The underlying structure of matter, however, is not mechanical.

Bohm thus suggests that thought processes and quantum systems have in common a certain ontological holism, which means that they cannot be analyzed too much in terms of distinct elements. This is so, he suggests, because an analysis beyond a certain point changes the "intrinsic nature" of the element in question. Elements can have certain characteristic properties (e.g., individual words have meaning; an individual electron exhibits either a wave or a particle nature), but they have such properties partly in virtue of the relations they have with other elements. Change those relations, and you may profoundly change the characteristic properties. For example, an electron that just exhibited a wave-like property may suddenly exhibit a particle-like property, if it is made to interact with an apparatus that measures its position. Analogously, in the spirit of Quine's semantic holism, a statement that previously seemed false may suddenly seem true if we make drastic enough adjustments elsewhere in the theoretical system it belongs to.

Bohm implies that the context-dependence of properties is no anomaly in a quantum universe. On the contrary, the context-dependence of properties seems to be a very fundamental feature of our physical universe. 
Although the context-dependence we find with e.g. meanings of words or statements is not necessarily the same type of context-dependence that we find with the properties of quantum systems, I would say that the similarities between thought/language and quantum systems in this respect are at least prima facie interesting and worth further exploration (cf. Stamenov 2004).

\subsection{The Classical Limit of Quantum Theory and the Logical Aspect of Thought Processes}

Bohm next points out yet another analogy, namely that between thought processes and the classical limit of quantum theory. Before discussing this analogy, let us briefly consider the physics side of the issue.

When we say that quantum theory has a classical limit, we acknowledge that, although at the fundamental quantum level movement seems discontinuous, there is a domain of physical phenomena where Newton's continuous and deterministic laws of motion provide an approximately correct description. Similarly, although quantum theory emphasizes the indivisible unity of the world, it seems that in our everyday experience we encounter a world that can, for all practical purposes, be analyzed into distinct elements. But how to reconcile the classical and quantum "worlds" - after all it seems that the world in which we live has both aspects? This question is connected with the correspondence principle that Bohr developed, described by Bohm (1951, p. 31) as follows:

this principle states that the laws of quantum physics must be so chosen that in the classical limit, where many quanta are involved, the quantum laws lead to the classical equations as an average. The problem of satisfying the correspondence principle is by no means trivial. In fact, the requirement of satisfying the correspondence principle, combined with indivisibility, the wave-particle duality, and incomplete determinism ... define $[\mathrm{s}]$ the quantum theory in an almost unique manner.

The discontinuous, indeterminate quantum level and the continuous, deterministic classical level are reconciled by noting that (p. 142)

... first, the discontinuities are too small to be seen on a classical level and, second, that so many quantum processes take place in any classical process that the deviation of the actual results from the statistical average is negligible.

Yet another quotation illustrates how to reconcile the unpredictability of an individual quantum with the causal laws on a macroscopic scale involving many such quanta (p. 30):

As for the appearance of apparently exact causal laws on a macroscopic scale, when only the probability of each elementary quantum 
transfer is determined, we merely note that, where many quanta are involved, the probability becomes almost a certainty (but not quite). This is very similar to the exact prediction, by insurance statistics, of the mean lifetime of a person within a large group, even though an exact prediction of the lifetime of a single individual in the group is not possible.

Having now a better idea of what is meant by the classical limit of the quantum theory, let us move on to explore what Bohm means when he says that there is "... a similarity between the thought process and the classical limit". His basic idea is that the "the logical process corresponds to the most general type of thought process as the classical limit corresponds to the most general quantum process" (pp. 169-170). This implies that the general structure of thought is analogous to the general structure of physical reality. In physical reality, as seen via the quantum theory, there is the level of general quantum processes with characteristic properties (indivisibility, wave-particle duality, uncontrollability, unpredictability etc.). Also, there is the classical limit where analysis into distinct elements is possible, as well as the mathematical description of the movement and interaction of these elements in terms of the deterministic laws of classical physics.

Bohm suggests that the relation between the logical process to the most general type of thought process is analogous to the relation between the classical limit and the most general quantum process. How does this analogy work? Bohm (1951, p. 170) writes:

In the logical process, we deal with classifications. These classifications are conceived as being completely separate but related by the rules of logic, which may be regarded as the analogue of the causal laws of classical physics. In any thought process, the component ideas are not separate but flow steadily and indivisibly. An attempt to analyze them into separate parts destroys or changes their meanings. Yet there are certain types of concepts, among which are those involving the classification of objects, in which we can, without producing any essential changes, neglect the indivisible and incompletely controllable connection with other ideas. Instead, the connection can be regarded as causal and following the rules of logic.

Bohm implies that there is a general type of thought process in which wholeness prevails. The component ideas are not separately existing elements with well-defined meanings. These ideas do not necessarily transform according to the rules of logic, but instead they "flow steadily and indivisibly". This general thought is a process, but not necessarily a process having order and necessity as characteristic of logical thought.

However, just as the physical world has a classically describable domain, so the process of thought includes the domain of logical thought 
process. In this "classical limit of thought" the indivisibility and uncontrollability of ideas that typically prevail in the general thought process have such a small effect that they can be neglected for all practical purposes. This makes it possible for relatively autonomous elements, for example concepts that classify objects, to arise. And it also makes possible for such elements to have causal relationships with each other, for example those causal relationships required for an actual volitional thinking process to proceed according to the rules of logic. In this way the "classical limit of thought", or the emergence of separate concepts and causal connections between them, makes logical thinking possible.

Of course, the classical limit is fundamentally important for both the physical world as we know it and for the very thought process that tries to have knowledge about the physical world. Bohm describes the role of the classical limit of both thought and quantum theory as follows (p. 170):

Logically definable concepts play the same fundamental role in abstract and precise thinking as do separable objects and phenomena in our customary description of the world. Without the development of logical thinking, we would have no clear way to express the results of our thinking, and no way to check its validity. Thus, just as life as we know it would be impossible if quantum theory did not have its present classical limit, thought as we know it would be impossible unless we could express its results in logical terms.

So it is important to note that Bohm does not deny the importance of the "classical limit of thought", any more than he would deny the importance of the classical limit of quantum theory. On the contrary, he emphasizes that logical thinking is fundamental for the enterprise of science, and for thought in general. But his approach implies that it would be a mistake to assume that logical thinking is the most general essence of the thought process, just as it would be a mistake to assume that classical physics reflects the essential nature of the physical world. He writes (p. 170):

Yet, the basic thinking process probably cannot be described as logical. For instance, many people have noted that a new idea often comes suddenly, after a long and unsuccessful search and without any apparent direct cause. We suggest that if the intermediate indivisible nonlogical steps occurring in an actual thought process are ignored, and if we restrict ourselves to a logical terminology, then the production of new ideas presents a strong analogy to a quantum jump. In a similar way, the actual concept of a quantum jump seems necessary in our procedure of describing a quantum system that is actually an indivisible whole in terms of words and concepts implying that it can be analyzed into distinct parts.

Bohm thus implies that the basic thinking process is "non-logical". Logical thinking emerges out of such a process under certain conditions, 
analogously to the way causal physical processes emerge out of the general quantum process at the classical limit. Interestingly, he suggests that the production of new ideas involves quantum-like thought procesess that essentially involve indivisible, non-logical steps. One central theme in the philosophy of science of the last century has been the difference between the context of discovery and the context of justification. Bohm gives a new expression to this idea when he implies that the discovery of new ideas may typically require a quantum-like, general, even non-logical thinking process, while their justification has to take place in the "classical limit of thought" and make use of the logical thinking process.

Notice also that Bohm's above quote implies that there is yet another feature which quantum processes and the general thought process have in common: it is difficult to talk about either of them when using the logical thinking process which employs well-defined concepts. He emphasizes that the notion of "quantum jump" is an example of a notion that we have to use in quantum physics when we try to talk about something indivisible in terms of words and concepts implying that it can be analyzed into distinct parts. Our customary ways of making sense of phenomena presuppose such analyzability; and when we apply analytical tools to phenomena that are strictly speaking unanalyzable, the result may be a somewhat artificial and unintelligible concept, such as that of a quantum jump.

\subsection{Thinking is Basically Quantum-Like}

The new proposal that comes out of Bohm's analogies is thus the idea that our thought process has a "quantum-like" aspect, and even more strongly, that the basic, most general type of thinking process is quantum-like. This basic thinking process is characteristic of quantumlike complementarity, in the sense that making one aspect of the process definite inevitably changes other equally significant aspects. It is also characteristic of quantum-like wholeness and relationality, in the sense that the characteristic properties (e.g., meaning) of elements of thought depend on indivisible connections with other elements. Further, the suggestion is that the way the general thought process changes from moment to moment is also quantum-like, for it involves non-logical steps. The component ideas in such a process are not separate but flow steadily and indivisibly. Finally, the basic thinking process seems to have a "classical limit", namely thinking in terms of well-defined concepts, including the logical thinking process.

What are the reasons that the most general type of thinking process is quantum-like? One possibility is that it is a mere co-incidence. But one can also ask whether the quantum-like features of the basic thinking process could be an indication that the physical aspect of the basic thinking process literally involves quantum processes. Considering this 
question, Bohm refers to Bohr's (1934) suggestion that "... thought involves such small amounts of energy that quantum-theoretical limitations play an essential role in determining its character". Bohm further writes (p. 170-171):

There is no question that observations show the presence of an enormous amount of mechanism in the brain, and that much of this mechanism must probably be regarded as operating on a classically describable level. In fact, nerve connections found thus far suggest combinations of telephone exchanges and calculating machines of a complexity that has probably never been dreamed of before.

Bohm thus acknowledges that a great deal of neural mechanisms is classically describable. But unlike contemporary cognitive neuroscientists who tend to assume that all neural mechanisms relevant to understanding cognition and consciousness are classically describable, Bohm follows Bohr and looks for a significant role for quantum processes in neural functioning (p. 171):

In addition to such classically describable mechanism that seems to act like a general system of communications, Bohr's suggestion involves the idea that certain key points controlling this mechanism (which are, in turn, affected by the actions of this mechanism) are so sensitive and delicately balanced that they must be described in an essentially quantum-mechanical way. (We might, for example, imagine that such key points exist at certain types of nerve junctions.) It cannot be stated too strongly that we are now on exceedingly speculative grounds.

There are by now a number of different and much more detailed suggestions about where such "quantum sites" could be located in the brain (see, e.g., Hameroff and Penrose 2014 or, for review, Atmanspacher 2011). What is important in Bohm's suggestion, however, is the general scheme. We are to envision two different levels of physical activity in the brain, one of them classically describable, while the other one needs to be described in a quantum theoretical way. This leads naturally to the assumption that the physical correlate of the logical thinking process is at the classically describable level of the brain, while the basic thinking process is at the quantum-theoretically describable level. Since Bohm implies that there is a two-way traffic between these two levels, a typical state of mind includes both of them.

It is also interesting to speculate that different types of states of mind could correspond to physiological states that differ with respect to the relative contribution made by "classical" and "quantum" neural processes. In some altered states of consciousness, for example, the classically describable neural processes might make a relatively small contribution to the content of experience, and as a result the holistic features typical of 
the quantum-theoretically describable neural processes would dominate. ${ }^{4}$ Although Bohm acknowledges that such ideas are speculative, he thinks that they are worthy of being pursued further (p. 171):

Bohr's hypothesis is not, however, in disagreement with anything that is now known. And the remarkable point-by-point analogy between the thought processes and quantum processes would suggest that a hypothesis relating these two may well turn out to be fruitful. If such a hypothesis could ever be verified, it would explain in a natural way a great many features of our thinking.

However, in the end we use the thinking process to explain the thinking process, so there is an intrinsic circularity to begin with. But the sort of thinking process that we commonly use in science is the logical thinking process. Thus, the logical thinking process tries to describe the most general thinking process. The logical thinking process is most suitable for describing the "classical limit" - be it the classical physical domain or the logical aspect of the thinking process. It is more difficult to describe quantum processes with the help of the logical thinking process - just as it is difficult to describe the general thinking process with it.

But we have already a fair amount of experience of dealing with quantum processes, both mathematically and conceptually. Now, if the general thinking process is analogous to quantum processes, we could make use of our experience with the quantum domain to try and explain the general thinking process. As an example, we saw above how Bohm characterized the production of new ideas as being analogous to a quantum jump.

Bohm also considers the alternative that the general thinking process does not literally involve quantum processes (p. 171):

Even if this hypothesis should be wrong, and even if we could describe the brains functions in terms of classical theory alone, the analogy between thought and quantum processes would still have important consequences: we would have what amounts to a classical system that provides a good analogy to quantum theory. At the least, this would be very instructive. It might, for example, give us a means for describing effects like those of the quantum theory in terms of hidden variables. (It would not, however, prove that such hidden variables exist).

Suppose that the general thought process is classically describable, and suppose that it is closely analogous to quantum processes. This opens

\footnotetext{
${ }^{4}$ The way I have here elaborated Bohm's early "quantum mind" ideas may seem very reductionist, as it is easy to interpret them as implying that thought processes can be reduced to a combination of classical and quantum processes in the brain. This may be how Bohm himself thought of the matter at the time. However, it is well known that his later overall mind-matter theory was not reductionist at all but rather a version of aspect monism (see Bohm 1980, 1990; Pylkkänen 2007).
} 
up the possibility that quantum processes might, after all, be classically describable; or at least it might be possible to describe them more fully than what standard quantum theory allows. ${ }^{5}$

\subsection{Understanding Quantum Theory from Understanding Thinking}

Another reason why Bohm thinks the analogy could be helpful, even in the absence of experimental data, is that it can give us a better feeling for quantum theory (p. 171):

For instance, suppose that we ask for a detailed description of how an electron is moving in a hydrogen atom when it is in a definite energy level. We can say that this is analogous to asking for a detailed description of what we are thinking about while we are reflecting on some definite subject. As soon as we begin to give this detailed description, we are no longer thinking about the subject in question, but are instead thinking about giving a detailed description. In a similar way, when the electron is moving with a definable trajectory, it simply can no longer be an electron that has a definite energy.

Here Bohm suggests to use the analogy between quantum processes and thought process as a tool that helps us to understand quantum theory. The kind of example he gives (asking someone to describe what they think) is, in principle, easily understandable to all of us. If such familiar features of the thought process are analogous to quantum processes, this makes it easier for us to understand quantum processes. Given that quantum theory is notoriously difficult to understand, this underlines the usefulness of the analogy.

But suppose that the thought process literally involves quantum processes. This would open up the possibility of yet another way for us to understand quantum processes (pp. 171-172):

If it should be true that the thought processes depend critically on quantum-mechanical elements in the brain, then we could say that the thought processes provide the same kind of direct experience of the effects of quantum theory that muscular forces provide for classical theory. Thus, for example, the pre-Galilean concepts of force, obtained from immediate experience with muscular forces, were correct, in general. ... We suggest that, similarly, the behavior

\footnotetext{
${ }^{5}$ I considered the above quote (which contains the reference to hidden variables) in a talk I gave at the University of Freiburg (at the interdisciplinary colloquium Visions in Research, April 23, 2014). In the discussion Harald Atmanspacher usefully pointed out that Bohm here refers to local hidden variables. These - unlike Bohm's own 1952 non-local hidden variable approach - have been ruled out by experiments like those of Aspect et al. (1982).
} 
of our thought process may perhaps reflect in an indirect way some of the quantum-mechanical aspects of the matter of which we are composed.

This is an extremely interesting possibility. Remember that quantum effects are often thought to lie in a domain that is not at all accessible to us in ordinary experience. As a consequence, it is assumed that we should not be surprised that it is difficult for us to understand the quantum domain - after all, we have no experience of it prior to the scientific experiments that probe this domain. Bohm suggests to turn this familiar scheme upside down. For it might be the case that all of us are, after all, familiar with some quantum-mechanical aspects of matter, in virtue of being familiar with an important part of ourselves, namely our thought processes! Quantum effects, which were supposed to lie in some mysterious domain that only physicists have access to, may lie much closer to home than we thought. If we are, psychologically, quantum-theoretical beings, then by being familiar with ourselves we may be familiar with quantum effects.

In philosophy, we sometimes speak about "maker's knowledge", implying that someone who has made or constructed something has a special kind of knowledge about it which others may lack. In a similar vein we might speak about "be-er's knowledge" - the knowledge someone has in virtue of being a certain kind of system (so I do not here mean to imply that a barley drink has knowledge!). ${ }^{6}$ Thought is an essential part of our being, and if thought processes reflect in an indirect way some of the quantum aspects of the matter of which we are composed, we might in principle have or be able to obtain "be-er's knowledge" of quantum aspects of matter.

The idea of "be-er's knowledge" figures prominently in philosophical discussions about conscious experience. For example, Nagel (1974) famously argued that there is something it is like to be a bat; and that we cannot find out what it is like just by studying the bat's brain and behavior. Only the "be-er" has direct experience of what it is like to be that system. In the case of the bat, it cannot communicate what it is like to be a bat in terms of concepts and logical thought to us.

For Nagel the example of the bat underlines the subjective, perspectivebound nature of consciousness. But even in the case of humans, it is not at all clear that we can communicate in any exhaustive sense in terms of concepts and logical arguments about what it is like to be a human being. The problem is not merely that a description of the objective, physiological correlates of conscious experience does not necessarily capture what it is like to be that system. There may be a part of our being that simply

\footnotetext{
${ }^{6}$ The physicist John Bell coined the term "beable" in this sense when in search for an ontological counterpart to the term "observable" in quantum theory.
} 
is so holistic, unpredictable etc. that it is difficult to capture in terms of conceptual and logical thought.

We can rephrase Bohm's suggestion by saying that part of what it is like to be a thinking human being is to have direct experience of the effects of quantum theory. Of course, this is not to say that we all thereby have conceptually organized knowledge about the effects of quantum theory. Bohm argues that the general thinking process is holistic and uncontrollable by its nature, and thus difficult to describe in terms of our usual scientific language that is organized in terms of separate concepts and logical arguments. Insofar as we are all familiar with the quantum-like general thinking process, our familiarity, acquaintance or understanding may be "pre-conceptual" and "pre-logical". ${ }^{7}$ We can, of course, try to improve this "pre-conceptual" familiarity or acquaintance and try to develop new concepts and principles to capture the holistic and uncontrollable aspects of our thought processes. We will return to the issue of pre-conceptual experience in Sec. 4.

In summary, we have seen how Bohm's analogies emphasize that the process of thought can be easily disturbed by introspective observation; that there is a limit in the extent to which significance of elements of thoughts can be analyzed; and that besides this generally incontrollable, unpredictable and indivisible character, the mind also has a domain of separable concepts which can be connected causally, following the rules of logic. Bohm further suggested that the analogies would get a natural explanation if it turned out that the neural processes that realize thought processes in the brain would involve quantum processes.

I hope that my extended presentation and discussion of Bohm's analogies has given the reader an example of what can be meant by the idea that was raised in the introduction of this paper: A new paradigm can, by analogy, function to draw attention to new aspects of the mind in introspection. The fairly new idea contained in these analogies is the suggestion that our general thinking process is quantum-like, whether or not this is a mere coincidence or the result of underlying quantum-physical correlates of thought. ${ }^{8}$ Although my discussion is admittedly sketchy and speculative, one should realize that the scientific and philosophical implications of this line of thought are potentially very significant. There is a possibility of a revolution in our understanding of the mind that might parallel the significance of the quantum revolution in physics.

\footnotetext{
${ }^{7}$ Compare Pylkkö (1998), and compare also the discussion of non-conceptual content (Bermúdez and Cahen 2011, Feil and Atmanspacher 2010). It might also be useful to consider the relation of the above to Russell's idea of knowledge by acquaintance (Russell 1912).

${ }^{8}$ It is interesting to consider to what extent Bohm's 1951 discussion anticipates the current significant advances in quantum cognition. One thinks here, for example, about Bruza et al.'s (2009) idea of a quantum-like human mental lexicon, or Aerts's (2009) notions of quantum-like concepts and the two modes of human thought.
} 
Let us now move on to briefly compare Bohm's approach with some other descriptions of inner experience and thought in contemporary philosophy of mind and cognitive science. ${ }^{9}$

\section{Cognitive Science and Quantum Analogies}

Traditional cognitive science was for a long time dominated by the so-called symbolic paradigm in which cognition was assumed to be mechanical symbol manipulation according to a set of rules or a "program". This resembles the domain of separable concepts in Bohm's description. Within cognitive science the symbolic paradigm was subjected to heavy criticisms, and as is well known, connectionist modeling was offered as an alternative way to describe cognition. ${ }^{10}$ Yet the advocates of the symbolic paradigm, most notably Fodor and Pylyshyn (1988), argued that connectionism lacks certain important features of the symbolic paradigm.

As an interesting attempt to reconcile the tension between connectionism and the symbolic paradigm, Smolensky (1988) proposed that the relation between them is analogous to the relation between quantum theory and classical mechanics. The idea is that a theory typically works in its proper domain, and that often a more general theory that applies in a broader domain can give rise to another theory that applies in a narrower domain as a limiting case. According to Smolensky's proposal we ought to view connectionism as the more general theory, describing a subsymbolic level of the mind, while the symbolic paradigm can be seen as a special, limiting case, describing those aspects of the mind where rule following and symbol manipulation seem to take place.

\footnotetext{
${ }^{9}$ One should not forget relations to psychology. In an insightful paper on introspection, Marcel (2003) makes some points similar to this paper, and develops them in more detail. Consider, for example, the following: "Attention can influence its object. Attending to one's experience, introspecting, changes the content, nature and form of the experience. It is also widely accepted that the content, nature and form of the experience that constitutes the content of awareness depends on the way that we attend" (Marcel 2003, p. 179). Marcel further provides an interesting discussion of the way (a) attention can be directed to components or to a whole; (b) how one's stance toward the object of attention can vary between immersion and detachment; (c) how attention can create its object; (d) how awareness distorts its object; and (e) how our theories can mask our experience. I think the way Marcel's ideas stand out as radical ideas is an indication that the sorts of features of the mind that Bohm's analogies raise are not that commonly noticed and acknowledged in contemporary psychology. For example, Marcel (2003, p. 179) writes: "John Lambie and I (2002) have recently emphasized what we call the mode of attention, the manner in which one attends at any time an aspect of attention stressed by William James (1890) but largely ignored by most current psychology."

${ }^{10}$ In recent years dynamical modeling of cognition has become important and can thus be seen as a third approach alongside symbolicism and connectionism. For an interesting recent discussion of these approaches see Eliasmith (2003).
} 
Smolensky's analogy is in some ways similar to Bohm's analogy. Both recognize that the mind has a "classical limit", as it were, a domain we can describe in terms of separable symbols, related in definite ways. And both agree that another, more general description is required to give a fuller description of the mind, and that the relation between the two descriptions is analogous to the relation between quantum theory and classical physics.

However, there are also differences between the two analogies. A particularly important difference is that Bohm's analogy suggests that cognition is more radically holistic, unpredictable and uncontrollable than what Smolensky's approach implies. The reason for this is that Bohm proposes a close analogy between quantum processes and processes of thought $d i$ rectly, whereas Smolensky makes a more methodological analogy between the relation of quantum and classical physics on the one hand, and of connectionism and the symbolic paradigm on the other.

Smolensky proposes that important, general features of cognition can be captured by connectionist networks. Connectionist networks have some holistic properties, and the mathematical formalism of connectionism has similarities to the mathematical formalism of quantum theory (see Perus 1995). However, it has been emphasized that traditional connectionist models do not go beyond the symbolic paradigm when it comes to mechanical computability. Pylkkö (1998, p. 94), for example, has suggested that "... most probably, all existing artificial neural networks and artificial models of chaotic systems are Turing-computable and, therefore, mechanical, in the obvious sense of the word". Thus the idea that cognition can be described in terms of connectionist models is not as different from the ideas of the symbolic paradigm as the proponents of connectionism, including Smolensky, seem to assume.

In contrast, Bohm's claim that our inner experiences and thought processes are closely analogous to quantum processes constitutes a much more radical break from the symbolic paradigm. It implies that mental processes have radically uncontrollable, indeterministic and semantically holistic features which cannot be adequately modelled by either the symbolic or the connectionist paradigms insofar as these are embedded in the framework of classical physics. Bohm's use of quantum analogies thus led him already in 1951 to propose an outline of a much more radical view of the mind than what cognitive science, inspired by the mechanistic computational models of the symbolic and connectionist type, came up with during the 1980s.

Now, the fact that Bohm's view of the mind is more radically holistic than that of either symbolic or connectionist cognitive science does not mean that it is correct. But how can we decide which view is correct? Perhaps we ought to listen to what the introspectionists and the phenomenologists have to say about the mind, then combine this with relevant computational models and empirical research in cognitive neu- 
roscience and then make our judgement. But this brings us back to our starting point. If introspection, including the introspection used in phenomenology, is theory-laden, this entails a tendency that introspection or phenomenology will, in a self-serving way, produce evidence to the view of the mind that the practitioner of introspection already had - consciously or unconsciously - before the introspection. For example, Pylkkö (1998, p. 80) writes:

... classical phenomenology (say, as it is developed in Husserl's Ideen 1913/1976) is not free of the intellectualist bias of the scientifictechnological attitude because classical phenomenology clearly sides with the conscious subject and its allegedly autonomous rationality and quite openly acknowledges the rational subject's right to dominate the rest of the mind.

In particular, introspectionists and phenomenologists not familiar with quantum physics are unlikely to suggest that the kind of unpredictability, uncontrollability and indivisibility they may encounter in introspection has the radical, non-classical character of quantum processes, simply because they are likely to lack the conceptual tools required to recognize such features, and to evaluate their difference from more classical-type features. Of course, all this does not prove that mind has quantum-like aspects. Perhaps an introspecter equipped with a quantum paradigm, such as Bohm, will be likewise biased in their introspection so that they attribute quantum-like behavior to aspects of mind that do not really call for it.

Nevertheless, it is at least in principle possible that, say, a quantum paradigm will provide a more adequate characterization of the mind than a mechanistic paradigm. In other words, human inner experience and mental processes may have certain features which are not completely determined by the mechanistic paradigm, and which different paradigms manage to deal with in varying degree of success. What any paradigm does is then to focus our attention in a particular way - so that we "see" certain aspects of what is "there" (in some sense "given") in experience.

We know we have been warned about the dangers of the myth of the given, most notably by Sellars (1956). But doing philosophy or science without the possibility of there being anything given in experience may also turn out to be a futile enterprise (cf. Westerlund 2014). I suggest for phenomenology it is better to have a classical plus quantum paradigm, instead of just a classical one. This way one may be able to "see" more of the conscious experience and thought processes - especially their unpredictable and holistic features, the sorts of features that are difficult to conceptualize. And the idea is that a richer and more inclusive paradigm can reveal further features. I do not claim that this suggestion is unproblematic, but I will not attempt to argue for it here. 
One might also note here that the connection between physics and the nature of human experience is an interesting theme in the history of philosophy. Most notably, Kant assumed that human experience necessarily has to exhibit certain features of Newtonian physics, such as spatiality, temporality and causality (see Kant 1787/1991, Strawson 1966). With his analogies Bohm draws attention to the non-Newtonian features of human experience, and in this sense he broadens the Kantian notion of what kinds of human experience are conceivable and possible. Kant was not wrong in claiming that human experience has Newtonian features, but he was perhaps wrong in his estimation of the limits within which human experience can vary.

Today, armed with the resources of post-Newtonian physics, we are in a position to see new analogies between physics and human experience. Consequently, we are encouraged to articulate our view of the limits within which human experience can vary in a new, broader way. Post-Newtonian physics inspires a post-Kantian view of human experience.

\section{Post-Phenomenology and Quantum Analogies}

Bohm is clearly not alone in suggesting that human inner experience, especially conscious thought has unpredictable, uncontrollable, indivisible and non-logical features. In particular, the philosophical movement some call "post-phenomenology" emphasizes such features. The connection between post-phenomenological ideas and quantum physics has been noticed and emphasized by, e.g., Plotnitsky (1994, 2002), Pylkkö (1998) and Globus (1995, 2003), who also emphasizes the role of quantum brain dynamics developed by Jibu and Yasue (1995), as well as Vitiello (2001). I have found Pylkkö's views particularly helpful when trying to make sense of Bohm's 1951 analogies to cognitive science and philosophy of mind.

Pylkkö developed a radical philosophical view that differs in some important ways from Bohm's philosophy of nature. In particular, Pylkkö advocates an antirealist view, in which one does not assume physical reality to exist in a well-defined way independently of human experience. Of primary significance for him is aconceptual experience, without any sharp division between concepts and objects. How do concepts and objects emerge then?

Pylkkö's idea is that they arise simultaneously as aconceptual experience divides itself into two aspects, concepts and the corresponding objects. However, he does not give too strong an ontological status for either the objects or the concepts. In contrast, Bohm had a tendency to defend a realist viewpoint in his natural philosophy, although his realism got increasingly modified, weakened and problematized as he kept on developing the epistemic implications of his holistic and processual view of 
nature (see Bohm 1980, Chap. 3). Here is a more detailed characterization of aconceptual experience given by Pylkkö (1998, p. 13) himself:

Let us take immediate and unstructured primitive experience as our philosophical starting point. This unarticulated and prelogical experience which we call aconceptual is what mind and language primarily is. It is not yet organized by concepts. Because we associate subjectivity strongly with the conceptual organization of experience we say also that, in aconceptual experience, there is no such hierarchy and perspective which characterize the subject's presence. The experience is, so to speak, holistically everywhere, without center, or it has a center which is not yet fully organized.

Viewed from the perspective of Pylkkö's post-phenomenology, Bohm's 1951 analogies clearly draw attention to some important aspects of the "aconceptual mind". For as we saw above, according to Bohm the general thinking process is non-logical, uncontrollable, unpredictable, and its semantic elements are indivisible in a way that makes it difficult to analyze it in conceptual terms. It seems to me that this fits fairly well with the view of the mind a post-phenomenologist like Pylkkö advocates, as long as one bears in mind the difference between, say, Pylkkö and Bohm on the issue of realism. No doubt Pylkkö's view of the mind differs also in other important respects from that of Bohm, but I think it is fair to say that the use of quantum analogies helped Bohm to capture some important and neglected features of the mind, which are today described by post-phenomenology in a more sophisticated and elaborate way.

\section{Concluding Reflections}

A central theme of this paper is the following: If introspection is paradigm-laden and if quantum physics gives rise to a new paradigm, might then someone armed with this new paradigm see new features in introspection, and thus have new things to tell us about conscious experience and the mind more generally?

As an example, I considered some analogies which Bohm already in 1951 proposed to hold between quantum processes and inner experiences, especially thought processes. These analogies draw attention to certain quantum-like features of the most general type of thought process, such as uncontrollability, unpredictability, semantic indivisibility, inseparability, non-logicality and non-conceptuality, while also doing justice to the more "classical" features of the thought process such as semantic separability, conceptuality, logicality and causality. The analogies acknowledge that these classical features are indispensable in many ways, but they also underline the fundamentality of the quantum-like aspect, for example, for the production of new ideas. 
We have seen that Bohm's analogies are in some important respects similar to Smolensky's analogy that tries to reconcile symbolic and connectionist paradigms in cognitive science. But we have also emphasized that Bohm's view of the mind, guided by his quantum intuitions, is more radically holistic than that of Smolensky's hybrid cognitive science. We also pointed out that it is difficult to judge which view is correct. If introspection is indeed paradigm-laden, whose paradigm are we going to use when introspecting in order to decide which paradigm is correct? The possible paradigm-ladeness of introspection gives rise to a circularity that ought to make us careful in our judgements regarding the nature of human experience. Considering a somewhat similar problem in the context of Heidegger's account of the problem of phenomenality, Westerlund (2014, p. 507) makes the following reasonable proposal:

... we need to take seriously the fact that a phenomenological description can only arise and justify itself as a description of a particular kind of experience, whereby the scope and clarificatory force of the description is always bound to remain open and undetermined.

My suggestion is that it is worth further considering the idea that human inner experience, and the general type of thought process in particular, has some quantum-like features. This opens up the possibility of a less mechanical and in my view more accurate description of human experience than what, say, cognitive science currently offers. I also related the view of the mind implicit in Bohm's analogies to Pylkkö's post-phenomenological view of the mind as aconceptual experience, and saw a fairly good fit between some aspects of these views.

What lessons can we draw from our brief study? I think the above discussion provides tentative evidence that quantum physical intuitions can in fact help to introspect human experience in a new, productive way. Introspection seems to be theory-laden, but this need not be seen merely as an epistemic limitation. It should by now be clear that such theory-ladeness requires us to become much more cautious when making statements about the "nature of the mind". But it seems to be at least a reasonable possibility that new theories can help us to see the phenomena we are exploring in a new light. And a theory originally developed to deal with a particular domain may prove useful in a prima facie very different domain - prima facie, because there are currently a number of different hypotheses proposing that in one relevant way or the other, the physical correlates of mental processes literally involve quantum processes. If this is correct, it would make it less of a puzzle how quantum theory may turn out to be relevant to describing the mind. In other words, two prima facie very different domains may turn out to be partly the same.

I recognize that it sounds somewhat far-fetched to many scientists that the study of physics could help us to understand the mind. But note 
that such a possibility is implicit in the whole program of philosophical atomism that underlies many of the successes of modern science. The traditional idea has been that physics studies the simple constituents of nature and the regularities in their behavior. This understanding of the behavior at the micro-level may then help us to understand features of more complex, higher-level systems. With quantum physics, however, the whole scheme of philosophical atomism is challenged, and one is forced to consider some radically holistic basic principles. Bohm refers to those principles when he suggests analogies between quantum processes and the mind. But surely, if the mind has such holistic features, it has had them all along, so does it really take quantum physics to notice and theorize about them?

The mechanistic view of the mind that dominates contemporary cognitive science and philosophy of mind - which is basically still nothing more than a hypothesis - has got part of its legitimacy and plausibility from the successes of the mechanistic view in physics and biology. In a similar vein, a radically holistic physics raises the possibility of a holistic biology and a holistic psychology (cf. Gierer 2002). Bohm's analogies suggest that just as the physical world has two aspects - the general holistic "quantum world", and as a special case, the mechanistic "classical world", so the human mind has two analogous aspects, the holistic general thinking process and the more mechanical, logical thinking process. Quantum physics can play an important role for psychology in suggesting a simple prototype of how a general holistic level and a special case of a mechanistic level can be reconciled. Thus, although the holistic aspects of the mind can no doubt be discovered without quantum physics (and indeed have been), I suggest that the quantum analogies can enrich both our introspective experience of the mind and the theories we construct in psychology and cognitive science.

Bohm's 1951 analogies are only a limited illustration of the way quantum physics can be useful when trying to understand the mind. For one thing, Bohm himself went on to interpret quantum physics in different ways and, not surprisingly, invented new analogies between interpretations of quantum physics and the mind. For example, Bohm and Hiley's "ontological interpretation" of quantum theory suggests that electrons are guided by a new type of field containing "active information" (Bohm and Hiley 1993). Bohm further suggested that the way such information acts is analogous to the way information acts in subjective human experience (see Bohm 1990, Pylkkänen 1992, 2007, Hiley and Pylkkänen 2005, Hiley 2004). His idea was that such active information could help us to understand what the mental and the physical sides of reality are and how they can affect each other. In this way, quantum theory can be used to tackle both the mind-body problem in general and the more specific problem of mental causation. 
In the context of his "implicate order" framework, Bohm (1980) likewise discussed the relation of mind and matter in a new way. The idea here is that mind and matter are analogous to non-locally connected quantum systems. They ought to be seen as correlated projections from a common multi-dimensional ground, rather than as separate substances in causal interaction. This is a radically new version of aspect monism, which again makes use of the resources of quantum theory.

It is important to note that Bohm's analogies differ from each other in important ways, although they have in common that they all originate from quantum physics (cf. Guarini 2003). This means that it is not a trivial task to construct a unified view of mind and matter on the basis of his analogies between quantum physics and the mind. For example, while the ontological interpretation (with its continuous particle trajectories) fits well with dynamical systems theory, the implicate order scheme challenges the latter. For the implicate order scheme underlines the discontinuity of movement and suggests that the basic mathematical structures needed to describe movement are algebras rather than differential calculus (Bohm 1980, Chap. 6; Pylkkänen 2007, pp. 117-122).

My suggestion is that the different Bohmian schemes, when applied to cognition and consciousness, can be seen as different tools which each can provide a useful way of looking at some aspect of the mind (cf. Murphy 1998). The implicate order scheme, I suggest ought to be seen as the more general and fundamental tool, but this need not exclude the use of the ontological interpretation scheme, as long as one remembers that the latter provides a more limited view. Whether or not these tools, and other similar tools developed by other researchers, help us to construct a more satisfactory theory of mind is currently an open question. But I hope that this study, which has focused on some of Bohm's very early ideas, illustrates some ways in which the consideration of quantum physics when studying the mind can be fruitful and open up radically new possibilities.

Perhaps some ideas discussed in this paper can also be useful when evaluating the relevance of quantum brain dynamics to the humanities, something that Globus $(1995,2003)$ in particular has emphasized. For example, Vitiello's (2001) focus on the "double" structure of the mind has been inspired by his consideration of dissipative quantum field theory. This, I think, constitutes yet another example of how quantum analogies can guide us in our search for a new and potentially richer view of the mind.

Notice that Vitiello's approach builds upon a more sophisticated scheme than quantum mechanics, namely quantum field theory. Applied to biological systems, this opens up the possibility of developing a more empirically accurate description of the physical aspects of the brain (Freeman and Vitiello 2006). At the same time, quantum field theory is often felt to be more difficult to understand than quantum mechanics. As a con- 
sequence, it can be more difficult to understand the analogies one might draw between quantum field theory and mental processes. I hope that the above consideration of some more simple analogies between thought processes and quantum processes can be helpful for a better understanding of the fascinating but difficult synthesis of quantum field theory, biology, neuroscience and (continental) philosophy of mind that is emerging from the work of Vitiello, Globus and others.

\section{Acknowledgments}

This is a modified version of a paper with the same title which was first published in the anthology Being and Brain. At the Boundary between Science, Philosophy, Language and Arts, ed. by G. Globus, K. Pribram and G. Vitiello, Advances in Consciousness Research 58, John Benjamins, Amsterdam 2004, pp. 165-195.

Part of this paper was written in October 2003 while I was a visiting scholar at the Department of Philosophy, Stanford University, based in the Metaphysics Research Lab at the Center for the Study of Language and Information. I would like to thank the director of the lab, Edward N. Zalta, for providing me a stimulating working environment. I also thank the various people who have commented on this paper. In particular, my colleague Stefan Berglund at the University of Skövde made some critical comments that prompted me to considerably develop an earlier draft. Of course, he is not to blame for any mistakes or unclarities that may remain! I am also grateful to Per Hansell and Gordon Globus for their comments and to Harald Atmanspacher for encouraging me to modify and re-publish the paper.

\section{References}

Aerts D. (2009) Quantum structure in cognition. Journal of Mathematical Psychology 53, 314-348.

Aspect A., Grangier P., and Roger G. (1982): Experimental test of Bell's inequalities using time-varying analyzers. Physical Review Letters 49, 1804-1807.

Atmanspacher H. (2011): Quantum approaches to consciousness. In The Stanford Encyclopedia of Philosophy, ed. by E.N. Zalta. Accessible at plato.stanford. edu/entries/qt-consciousness/.

Bermúdez J. and Cahen A. (2011): Non-conceptual mental content. In Stanford Encyclopedia of Philosophy, ed. by E.N. Zalta. Accessible at plato.stanford. edu/entries/content-nonconceptual/

Bohm D. (1951). Quantum Theory, Prentice-Hall, Englewood Cliffs.

Bohm D. (1980). Wholeness and the Implicate Order, Routledge, London. 
Bohm D. (1990). A new theory of the relation of mind and matter. Philosophical Psychology 3(2), 271-286.

Bohm D. and Hiley B.J. (1993): The Undivided Universe: An Ontological Interpretation of Quantum Theory, Routledge, London.

Bohr N. (1934): Atomic Theory and the Description of Nature, Cambridge University Press, London.

Bruza P., Kitto K., Nelson D. and McEvoy C. (2009): Is there something quantum-like about the human mental lexicon? Journal of Mathematical Psychology 53, 363-377.

Campbell N. (1957). Foundations of Science: The Philosophy of Theory and Experiment, Dover, New York.

Cushing J.T. (1990). Theory Construction and Selection in Modern Physics: The S-Matrix, Cambridge University Press, Cambridge.

Eliasmith C. (2003): Moving beyond metaphors: Understanding the mind for what it is. Journal of Philosophy 100, 493-520.

Feil D. and Atmanspacher H. (2010): Acategorial states in a representational theory of mental processes. Journal of Consciousness Studies 17(5-6), 72-101.

Feyerabend P.K. (1993): Against Method: Outline of an Anarchist Theory of Knowledge, Verso, London.

Fodor J. and Pylyshyn Z. (1988): Connectionism and cognitive architecture: A critical analysis. In Connections and Symbols, ed. by S. Pinker and J. Mehler, MIT Press, Cambridge, pp. 3-71.

Freeman W.J. and Vitiello G. (2006: Nonlinear brain dynamics as macroscopic manifestation of underlying many-body field dynamics. Physics of Life Reviews 3, 93-118.

Gierer A. (2002): Holistic biology - Back on stage? Comments on post-genomics in historical perspective. Philosophia Naturalis 39(1), 25-44.

Globus G. (1995): The Postmodern Brain, John Benjamins, Amsterdam.

Globus G. (2003): Quantum Closures and Disclosures, John Benjamins, Amsterdam.

Guarini M. (2003): Bohm's metaphors, causality and the quantum potential. Erkenntnis 59, 77-95.

Hameroff S. and Penrose R. (2014): Consciousness in the universe: A review of the orch OR theory. Physics of Life Reviews 11(1), 39-78. Article accessible at http://dx.doi.org/10.1016/j.plrev.2013.08.002.

Hanson N.R. (1958): Patterns of Discovery, Cambridge University Press, Cambridge.

Hesse M.B. (1966): Models and Analogies in Science, University of Notre Dame Press, Notre Dame.

Hesse M.B. (1974): The Structure of Scientific Inference, University of California Press, Berkeley. 
Hiley B.J. (2004) Information, quantum theory and the brain. In Brain and Being, ed. by G. Globus, K. Pribram, and G. Vitiello, John Benjamins, Amsterdam, pp. 199-216.

Hiley B.J. and Pylkkänen P. (2005): Can mind affect matter via active information? Mind and Matter 3(2), 7-26.

Husserl E. (1913/1976): Ideas. General Introduction to Pure Phenomenology, transl. by W.R. Royce Gibson, George Allen and Unwin, London.

James W. (1890): The Principles of Psychology, Harvard University Press, Cambridge.

Jibu M. and Yasue K. (1995): Quantum Brain Dynamics and Consciousness, John Benjamins, Amsterdam.

Jones W.T. (1975): A History of Western Philosophy. The Twentieth Century to Wittgenstein and Sartre, Harcourt Brace Jovanovich, New York.

Kant I. (1787/1991): Critique of Pure Reason, 2nd edition, originally published in German in 1787. J.M. Dent \& Sons Ltd., London.

Kuhn T.S. (1970): The Structure of Scientific Revolutions, University of Chicago Press, Chicago.

Ladyman J. and Ross D. (2007): Every Thing Must Go: Metaphysics Naturalized, Oxford University Press, Oxford.

Lambie J.A. and Marcel A.J. (2002): Consciousness and emotion experience: A theoretical framework. Psychological Review 109, 219-259.

Marcel A.J. (2003): Introspective report: Trust, self-knowledge and science. Journal of Consciousness Studies 10(9-10), 167-186.

Murphy T.S. (1998): Quantum ontology. In Deleuze and Guattari - New Mappings in Politics, Philosophy and Culture, ed. by E. Kaufman and K.J. Heller, University of Minnesota Press, Minneapolis, pp. 211-229.

Nagel T. (1974): What is it like to be a bat? Philosophical Review 83, 435-450.

Penrose R. (1994): Shadows of the Mind, Oxford University Press, Oxford.

Perus M. (1995): Analogies of the quantum and neural processing Consequences for cognitive science. In New Directions in Cognitive Science, ed. by P. Pylkkänen and P. Pylkkö, Finnish Artificial Intelligence Society, Helsinki, pp. 115-123.

Pickering A. (1984). Constructing Quarks: A Sociological History of Particle Physics, Edinburgh University Press, Edinburgh.

Plotnitsky A. (1994): Complementarity. Anti-Epistemology after Bohr and Derrida, Duke University Press, Durham.

Plotnitsky A. (2002): The Knowable and the Unknowable. Modern Science, Nonclassical Thought, and the "Two Cultures", University of Michigan Press, Ann Arbor.

Plotnitsky A. (2010): Epistemology and Probability. Bohr, Heisenberg, Schrödinger and the Nature of Quantum-Theoretical Thinking, Springer, Berlin. 
Pylkkänen P. (1992): Mind, Matter and Active Information. The Relevance of David Bohm's Interpretation of Quantum Theory to Cognitive Science. Reports from the Department of Philosophy, University of Helsinki.

Pylkkänen P. (2007): Mind, Matter and the Implicate Order., Springer, Berlin. Pylkkö P. (1998): The Aconceptual Mind: Heideggerian Themes in Holistic Naturalism, John Benjamins, Amsterdam.

Quine W.V.O. (1951/1961): From a Logical Point of View, Harper \& Row, New York.

Revonsuo A. (2006): Inner Presence: Consciousness as a Biological Phenomenon, MIT Press, Cambridge.

Russell B. (1912): The Problems of Philosophy, Oxford University Press, Oxford.

Sellars W. (1956): Empiricism and the philosophy of mind. In Minnesota Studies in the Philosophy of Science, Vol. I, ed. by H. Feigl and M. Scriven, University of Minnesota Press, Minneapolis, pp. 253-329.

Shanon B. (1991): Cognitive psychology and modern physics: Some analogies. European Journal of Cognitive Psychology 3(2), 201-234.

Smolensky P. (1988): On the proper treatment of connectionism. Behavioral and Brain Sciences 11, 1-74.

Stamenov M. (2004): The rheomode of language of David Bohm as a way to re-construct the acess to physical reality. In Brain and Being, ed. by G. Globus, K. Pribram, and G. Vitiello, John Benjamins, Amsterdam, pp. 147-164.

Strawson P.F. (1966): The Bounds of Sense. An Essay on Kant's Critique of Pure Reason, Routledge, London.

Sundqvist F. (2003): Perceptual Dynamics: Theoretical Foundations and Philosophical Implications of Gestalt Psychology, Acta Philosophica Gothoburgensia, Göteborg.

Van Gulick R. (1995): What would count as explaining consciousness? In Conscious Experience, ed. by T. Metzinger, Imprint Academic, Exeter.

Van Loocke P., ed. (2001): The Physical Nature of Consciousness, John Benjamins, Amsterdam.

Vitiello G. (2001): My Double Unveiled. The Dissipative Quantum Model of Brain, John Benjamins, Amsterdam.

Westerlund F. (2014): Heidegger and the Problem of Phenomenality, Philosophical Studies from the University of Helsinki. Full text accessible at helda. helsinki.fi/bitstream/handle/10138/45258/Westerlund_vaitoskirja.pdf? sequence $=1$. 